\title{
Stochastic reversal dynamics of two interacting magnetic dipoles: a simple model experiment
}

\author{
Nicolas Plihon ${ }^{1}$, Mickael Bourgoin ${ }^{1}$, and Jean-François Pinton ${ }^{1}$ \\ 1 Univ Lyon, Ens de Lyon, Univ Claude Bernard, \\ CNRS, Laboratoire de Physique, F-69342 Lyon, France \\ Sophie Miralles ${ }^{1,2}$ \\ 1 Univ Lyon, Ens de Lyon, Univ Claude Bernard, \\ CNRS, Laboratoire de Physique, F-69342 Lyon, France \\ ${ }^{2}$ Laboratoire des Ecoulements Géophysiques et lndustriels LEGI, \\ Université Grenoble Alpes 38058 GRENOBLE CEDEX 9
}

(Dated: December 8, 2019)

\begin{abstract}
We report an experimental study of the dynamics of two coupled magnetic dipoles. The experiment consists in two coplanar permanent disk magnets separated by a distance $d$, each allowed to rotate on fixed parallel axis - each magnets axis being perpendicular to its dipolar moment vector. A torque of adjustable strength can be externally applied to one of the magnets, the other magnet being free. The driving torque may be time independent or temporally fluctuating. We study the influence of the parameters of the driving torque on the dynamics of the coupled system, in particular the emergence of dynamical regimes like stochastic reversals. We report transitions between stationary and stochastic reversal regimes. All the observed features can be understood by a simple mechanical dynamical model. The transition between statistically stationary regimes and reversals is explained introducing an effective potential energy incorporating both the coupling between magnets and the external driving. Relations between this simple experimental model with macroscopic models of magnetic spins coupling, as well as with chaotic reversals of turbulent dynamos are discussed.
\end{abstract}

\section{INTRODUCTION}

Multi-stable stochastic processes play a crucial role in many fundamental problems in all fields of science (physics, chemistry, geophysics, biology, etc.) [1]. In such processes a dynamical system can transit between stable deterministic states separated by an energy barrier; the transition is generally activated by natural or external fluctuations (stochastic noise) promoting the escape of the system from one state to the other. The investigation of the probability of such escape events is a classical and complex problem (known as Kramers problem [2]), and an active research field of multi-stable stochastic systems. It depends on many aspects, such as the amplitude of the energy barrier between stable states, the type of activating noise (additive, multiplicative), the correlation time and the colour of the noise. In particular, multi-stable stochastic systems are omnipresent in spin systems or magnetic dipole-dipole interaction. For instance, finite size ensembles of magnetic dipoles exhibit transitions between ferromagnetic phases and chaotic regimes (zero average magnetisation) at zero temperature as shown in [3], the transition being dependent on the number of dipoles and on the strength of the coupling [4]. Similar transitions are often observed in fluid mechanics systems. For instance, closed turbulent flows generated between counter-rotating impellers (so called von Kármán flows) are known to exhibit hysteresis and multi-stability [5] with transitions between states activated by turbulent fluctuations. Recent experiments by de la Torre \& Burguete [6] have shown for instance that a perfectly sym- metric von Kármán flow can exhibit such transitions between two asymmetric flow configurations (the global symmetry being then statistically recovered). Another example is given by the long term Lagrangian dynamics of a large particle in symmetric von Kármán flows [7], which have been shown to be preferentially trapped in two stable attractors (in the close vicinity of each impeller), with rare and sudden transitions between both sides of the flow activated by turbulent fluctuations. Such transitions have also been reported in dynamo experiments. In these systems involving electrically conducting fluids, an instability converting kinetic energy into magnetic energy is at the origin of the growth of a large-scale magnetic field. Reversals between two opposite polarities of the magnetic field have for instance been observed in the homogeneous von Kármán Sodium (VKS) experiment [8], as well in the semi-synthetic Bullard-von Kármán dynamo setup [9].

The present study explores the possibility to revisit some generic properties of the influence of noise on such multistable systems by considering a simple table-top experiment with two coupled magnetic dipoles. The free dynamics of two coupled, identical, frictionless magnetic dipoles has already been described by [10] with analytical solutions depending on initial conditions. We consider here a similar geometry but with coupled dipoles having distinct moments of inertia and we incorporate losses. Moreover, in the present study, the angular position of one of the dipoles is forced by a driving torque (which could be the realization of a stochastic noise), the other dipole being free. The study is in the same vein 
as the one reported in [11] which highlighted magnetic frustration at a macroscopic scale, with an ensemble of macroscopic magnetic dipoles. The article is organised as follows: first, in section 2, we describe the setup of the table-top experiment. In section 3, the computation of the fixed points of the system are detailed. The different dynamical regimes obtained for specific parameters are explained in section 4 and a phenomenological model is given in section 5 to explain qualitatively the observed regimes. Arguments are given in section 6 to compare our model experiment with the dynamical regimes observed in dynamo experiments. In particular, our tabletop experiment shares similar statistical properties with the reversals observed in the VKS experiment, such as transitions between stationary dynamos and reversals, distribution of the waiting time between reversals, as well as the relative influence of the noise on the dynamics.

\section{EXPERIMENTAL SETUP}

The experimental setup is schematically displayed in figure 1. Two Neodynum-Iron-Bore dipolar magnets are fixed at the tip of two rotating parallel shafts. These magnets are very thin circular plates (of diameter 30 $\mathrm{mm}$, thickness $1 \mathrm{~mm}$ ) magnetised along one diameter, with dipolar moments $\mathcal{M}_{1}$ and $\mathcal{M}_{2}$ respectively. Both magnets are set in the same vertical plane $(x, y)$ (perpendicular to their rotation axis) and separated by a distance $d$. Note that the distance $d$ sets the amplitude of the coupling between the two magnets and can be modified at will. The angular positions of each dipolar magnet are denoted $\theta_{1}$ and $\theta_{2}$, relative to the reference horizontal axis $x$ (see figure 1-(a)). When magnets are left interacting without external driving, the stable fixed points are $\theta_{1}=\theta_{2}=0(\bmod \pi)$ and the unstable fixed points are $\theta_{i}= \pm \pi / 2(\bmod \pi)$. When an external torque is applied to shaft 1 such that $\theta_{1}>0$, it leads to a stable equilibrium angle $\theta_{2}<0$, as displayed in figure 1. Such an external torque is applied by a magnetic driving system added to the opposite end of shaft 1 (on which $\mathcal{M}_{1}$ is set) - the magnetic nature of the drive was designed to avoid additional mechanical friction. The driving system is also schematically displayed in figure 1 and consists of a quadrupolar stator/rotorlike system. The rotor is composed of small permanent magnets while the stator is composed of eight coils. The currents in these coils impose the strength of the maximum torque applied to shaft 1 as well as preferential orientations for $\theta_{1}$. This is achieved by driving currents $I_{i}$ in coil $i$ as $I_{1}=I_{5}=-I_{3}=-I_{7}=I_{0} \cos \alpha$ and $I_{2}=I_{6}=-I_{4}=-I_{8}=I_{0} \sin \alpha$ : the maximum strength of the externally applied torque is thus proportional to $I_{0}$ and the preferential orientation of $\theta_{1}$ is $\alpha / 2(\bmod \pi)$ (reached when the driving torque overcomes the coupling between the magnets). Figure 1 displays the situation with $\alpha=\pi / 2$ (with the external driving torque dominating over the dipolar magnets interaction) for which $\theta_{1}=\alpha / 2=\pi / 4$. The choice of this quadrupolar forcing has been favored since (i) the strength and direction of the driving torques can be independently set, (ii) the modulus $\pi$ of fixed points is preserved (see next section). Note that both $I_{0}$ and $\alpha$ may explicitly depend on time.

The present study focuses on the experimental observation of the relative influence of the coupling between the two magnets and of the external driving torque on the dynamics of the system - the coupling being controlled by the distance $d$ for a fixed couple of magnets and the driving by the current in the external coils (both the amplitude $I_{0}$ and the angle $\alpha$ ).

(a)
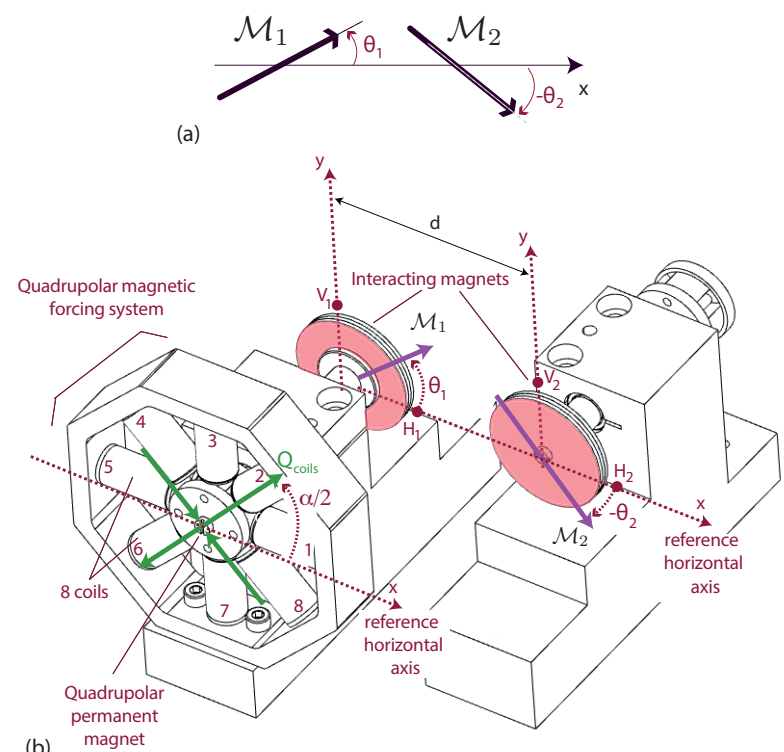

FIG. 1. (Color online) (a) 2D schematic view of the interaction of the two magnets studied in this article (b) Schematic of the experimental setup

Currents in the coils are driven by two bipolar current amplifiers Maxon LSC30/2, feeding the odd and even coils up to $I_{0}^{\max }=2 \mathrm{~A}$. These amplifiers are controlled by analog outputs of a National Instruments PCIe 6321 digitised at $400 \mathrm{~Hz}$. Around each dipolar magnets, the projections of the magnetic field created by the dipolar moments $\mathcal{M}_{1}$ and $\mathcal{M}_{2}$ along the $x$ horizontal axis (at locations $H_{1}$ and $H_{2}$ ) and along the $y$ vertical axis (at locations $V_{1}$ and $V_{2}$ ) are measured using AnalogDevices 22151 Hall effect sensors, sampled at a rate of $400 \mathrm{~Hz}$ by National Instruments PCIe 6321 analog digitisers. The angular positions $\theta_{1}$ and $\theta_{2}$ of the interacting magnets are then computed from these measurements after a calibration procedure. Details about the experiment are provided as in appendix A.

The dynamics of the system is described by a set of 2 coupled differential equations (see appendix A for de- 
tailed calculations) in their normalised form:

$$
\begin{aligned}
J_{1}^{*} \frac{d^{2} \theta_{1}}{d t^{2}}= & \mathcal{A} \sin \left(\alpha-2 \theta_{1}\right)-\sin \theta_{2} \cos \theta_{1}-2 \sin \theta_{1} \cos \theta_{2} \\
& -F_{1}^{*} \frac{d \theta_{1}}{d t}-K_{1}^{*} \operatorname{sign}\left(\frac{d \theta_{1}}{d t}\right) \\
J_{2}^{*} \frac{d^{2} \theta_{2}}{d t^{2}}= & -\sin \theta_{1} \cos \theta_{2}-2 \sin \theta_{2} \cos \theta_{1} \\
& -F_{2}^{*} \frac{d \theta_{2}}{d t}-K_{2}^{*} \operatorname{sign}\left(\frac{d \theta_{2}}{d t}\right)
\end{aligned}
$$

with $J_{i}^{*}, F_{i}^{*}$ and $K_{i}^{*}$ respectively denoting the moments of inertia of shaft $i$, the fluid friction coefficient and the solid friction coefficient acting on shaft $i$, normalised by the coupling strength $\mu_{0} \mathcal{M}_{1} \mathcal{M}_{2} / 4 \pi d^{3}$. Introducing $D$ as an effective driving efficiency of the magnetic drive, $\mathcal{A}$ denotes the normalised driving torque as $\frac{D I_{0}}{\mu_{0} \mathcal{M}_{1} \mathcal{M}_{2} / 4 \pi d^{3}}$ - in other words $\mathcal{A}$ is the ratio between the maximum driving torque $D I_{0}$ and the coupling between the two magnets.

When the system is uncoupled (infinite $d$ ) or when one of the shafts is kept static, the dynamics simplifies as a second order differential equation with damping. In that case, each of the parameters of equations A1 and A2 can be determined by fitting experimental data with a second order model system. These measurements are straightforward and are described in appendix B.

\section{COMPUTATION AND MEASUREMENT OF STATIONARY FIXED POINTS}

Stationary fixed points of the system (A1, A2) can be computed as solutions of the system:

$$
\begin{array}{r}
\frac{\mathcal{A}}{3} \sin \left(\alpha-2 \theta_{1}\right)+\sin \theta_{2} \cos \theta_{1}=0 \\
\sin \theta_{1} \cos \theta_{2}+2 \sin \theta_{2} \cos \theta_{1}=0
\end{array}
$$

and fixed points depend on the values of $\mathcal{A}$ (or equivalently on $I_{0}$ and $d$ ) and $\alpha$.

It is also instructive to investigate the nature of these stationary fixed points from an energy point of view. It is convenient to introduce an effective potential energy from which the conservative dynamics of the system can be derived. The interaction potential, normalised by $\mu_{0} \mathcal{M}_{1} \mathcal{M}_{2} / 4 \pi d^{3}$ reads:

$$
V_{i n t}=-3 \cos \theta_{1} \cos \theta_{2}+\cos \left(\theta_{2}-\theta_{1}\right),
$$

from which the normalised torque applied by magnet 2 on magnet 1 is computed as $\Gamma_{21}=-\frac{\partial V_{i n t}}{\partial \theta_{1}}$ and the normalised torque applied by magnet 1 on magnet 2 as $\Gamma_{12}=-\frac{\partial V_{i n t}}{\partial \theta_{2}}$

Equivalently, the normalised potential accounting for the driving torque applied to magnet 1 by the driving system reads:

$$
V_{\text {drive }}=-\frac{\mathcal{A}}{2} \cos \left(\alpha-2 \theta_{1}\right),
$$

from which the normalised driving torque acting on magnet 1 is computed $\Gamma_{\text {drive }}=-\frac{\partial V_{\text {drive }}}{\partial \theta_{1}}$. We thus introduce a normalised effective potential energy $V$ accounting for both the coupling between magnets and the external drive as:

$V=-3 \cos \theta_{1} \cos \theta_{2}+\cos \left(\theta_{2}-\theta_{1}\right)+\frac{\mathcal{A}}{2} \cos \left(\alpha-2 \theta_{1}\right)+C$

where the constant $C$ is adjusted for the minimum value of $V$ to be zero.

From system (3)-(4), it is readily checked that eight fixed points exist for $\left(\theta_{1}, \theta_{2}\right)$ and that if $\theta_{1}^{0}$ and $\theta_{2}^{0}$ are solution of the above system, then $\theta_{1}^{0}+\pi$ and $\theta_{2}^{0}+\pi$ are also solutions. The quadrupolar driving scheme thus preserves the modulus $\pi$ of the stationary fixed points. However, in the present setup, only four fixed points are easily accessible to the dynamics of the system. This can be understood by investigating the evolution of potential $V$ in the configuration space $\left(\theta_{1}, \theta_{2}\right)$ which is displayed in figure 2 without driving (i.e. $\mathcal{A}=0$ ). Two stable fixed points correspond to global minima of potential $V$ and are displayed as white circles $\left(\theta_{1}=\theta_{2}=0(\bmod 2 \pi)\right.$ and $\left.\theta_{1}=\theta_{2}= \pm \pi(\bmod 2 \pi)\right)$. The two reachable unstable fixed points, which are saddle points in the configuration space $\left(\theta_{1}, \theta_{2}\right)$, are displayed as white crosses. Four other unstable fixed points (either saddle points or global maxima) are displayed as black diamonds, with potential values respectively 3 and 4 (i.e. 3 and 4 times larger than the potential of the two saddle points displayed as white crosses). In the context of our study, the dynamics will be confined in the dark energy valley of figure 2 - along the diagonal $\theta_{2} \sim-\theta_{1}$ when $\mathcal{A} \sim 0$ - and the four fixed points at higher potential (black diamonds) are never observed (see figure 5). From the initial eight fixed points, only four of them (two stable and two unstable ones) are thus accessible. The situation remains similar when considering cases for which $\mathcal{A} \neq 0$; as shown below, the potential landscape is modified, however the dynamics is still confined to low energy valleys of the potential landscape.

Let us now focus on the stable fixed points in the more general situation for which $\alpha$, the preferential orientation of magnet 1 occurring from the driving torque, and $\mathcal{A}$, the strength of the driving torque, are non zero. When $\alpha \neq 0$, two asymptotic behaviours are identified:

- $\mathcal{A} \ll 1$ : when the coupling between the two interacting magnets dominates over the driving, $\theta_{1} \rightarrow 0$ $(\bmod \pi)$ (in the experiment, for vanishing values of $I_{0}$ and finite $d$ value).

- $\mathcal{A} \gg 1$ : when the driving dominates over the coupling between the two interacting magnets, $\theta_{1} \rightarrow$ 

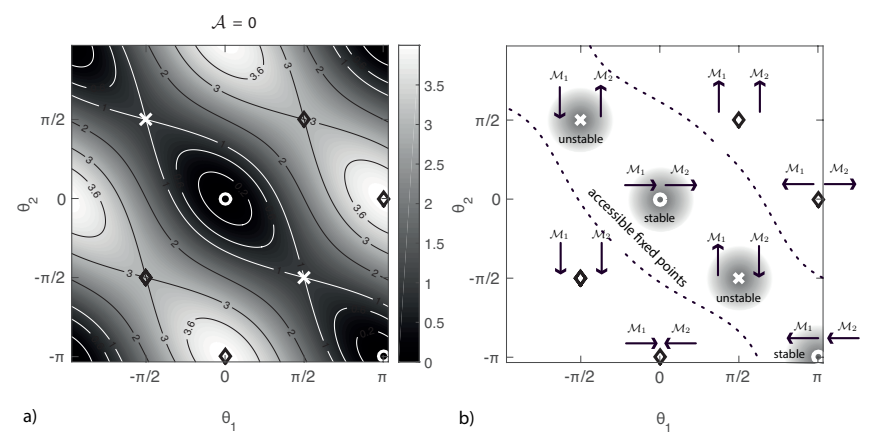

b)

$\theta_{1}$

FIG. 2. (a)Evolution of potential $V$ in the configuration space $\left(\theta_{1}, \theta_{2}\right)$ when $\mathcal{A}=0$; contour lines for $0.2,0.6,1,2$, 3 and 3.6 energy values. (b) Fixed points as in (a) and associated magnets $\mathcal{M}_{1}, \mathcal{M}_{2}$ configurations: stable fixed points (white circles), unstable saddle points (white crosses) and non-accessible unstable fixed points (black diamonds).

$\alpha / 2(\bmod \pi)$ (in the experiment, for large $I_{0}$ values at finite $d)$.

For a given value of the interaction length $(d=45 \mathrm{~mm})$, the stationary stable fixed points $\theta_{i}$ have been measured as a function of $\mathcal{A}$ and systematically compared to the solutions computed for system $(3,4)$ for several values of $\alpha$ - three of which are displayed in Figure 3. Experimental points are displayed as symbols together with the solutions of system $(3,4)$, displayed as full lines (black filled symbols and black full line for $\theta_{1}$ and open red symbols and red line for $\theta_{2}$ ). The agreement is very good, within the error bars of the experimental data. The asymptotic limits are clearly observed, especially for the largest values of $\alpha$. Note that the relation for fixed points according to equation 4 reads $\tan \theta_{1}=-2 \tan \theta_{2}$ since the driving torque is applied only on $\mathcal{M}_{1}$.

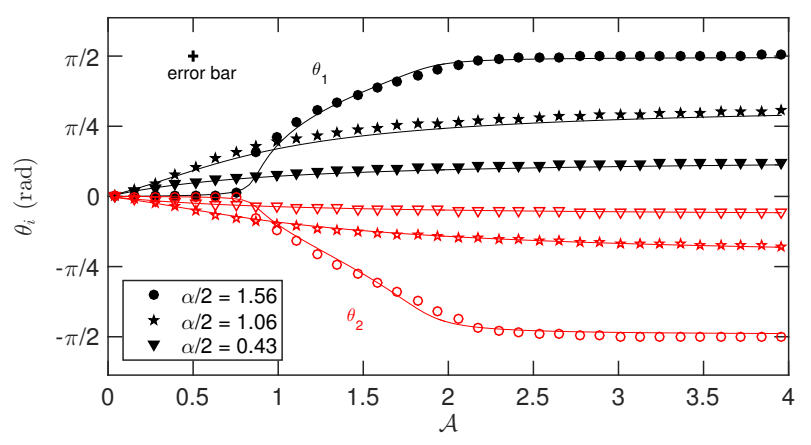

FIG. 3. Color online. Evolution of experimental and theoretical fixed points as a function of the normalised driving amplitude $\mathcal{A}$ for three values of $\alpha$ : $\theta_{1}$ (experiment: full black symbols) and $\theta_{2}$ (experiment: open red symbols).

This evolution of the stable fixed points as a function of $\mathcal{A}$ can also be understood from the evolution of the potential landscape $V\left(\theta_{1}, \theta_{2}\right)$. Figure 4 shows the evo- lution of of $V$ for 6 values of $\mathcal{A}$ and $\alpha / 2=1.56$ (corresponding to the experimental points black circles in figure 3). Both the amplitude of the potential and the shape of the potential landscape as a function of $\theta_{1}$ and $\theta_{2}$ are modified. In particular global minima are observed around $\theta_{1} \sim \theta_{2} \sim 0 \bmod (\pi)$ at low values of $\mathcal{A}$ (below 0.5 ), which correspond to the stable fixed points of figure 2. When $\mathcal{A}$ increases, a shift of the global minima towards $\theta_{1} \sim \alpha / 2(\bmod \pi)$ and $\tan \left(\theta_{2}\right)=-\tan \left(\theta_{1}\right) / 2$ is observed. In particular, for $\mathcal{A}>2$ in figure 4 , the energy landscape drastically changed as compared to the $\mathcal{A}=0$ case: the low energy valley is no more along the diagonal $\theta_{1} \sim-\theta_{2}$, but has shifted toward vertical lines around $\theta_{1} \sim \alpha / 2(\bmod \pi)$. The potential barrier between stable fixed points increases as $\mathcal{A}$ increases (note the colorscales in figure 4). This can be understood in the following way: when $\mathcal{A} \gg 1$, the driving torque is dominant and magnet 1 barely rotates around its axis even if magnet 2 rotates. In this configuration, the unstable fixed point with the lowest energy is now $\theta_{1} \sim \theta_{2}$; this unstable fixed point is now easily accessible to the system.
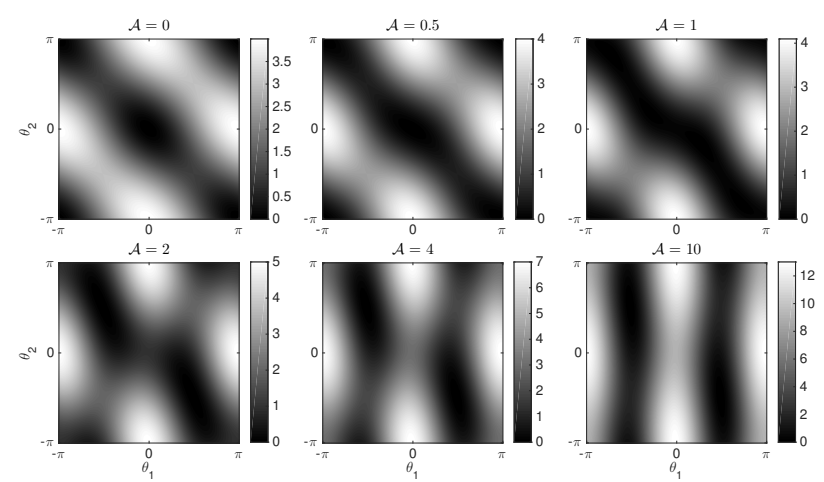

FIG. 4. Evolution of potential $V$ in the configuration space $\left(\theta_{1}, \theta_{2}\right)$, for increasing values of $\mathcal{A}$ from top left to bottom right and $\alpha / 2=1.56$.

\section{DYNAMICS WITH TIME-VARYING DRIVING}

Let us now investigate the dynamics of the coupled system (A1,A2) with a time-varying driving. Several choices may be made for the time-dependent variation of the driving torque through time-variation of the driving current $I_{0}$ or the driving angle $\alpha$. In the remaining of the paper $\alpha$ is kept constant and time-dependence arises from the amplitude of the driving current $I_{0}$ - or equivalently $\mathcal{A}$ at constant $d$. Since similar dynamics have been obtained for time-dependent phase and/or timedependent magnitude of the driving torque, we will focus on cases with time dependent magnitude of the driving torque without any loss of generality. $\mathcal{A}(t)$ was chosen as the digital realisation of a delta-correlated gaussian process, with average $\langle\mathcal{A}\rangle$ and standard deviation $\tilde{\mathcal{A}}$. This 
choice was motivated by the observation of non-trivial dynamics even in the presence of such simple noise realisations. The choice of the nature of the noise is further discussed in the conclusion, in particular when discussing the possible application of this analogue model to turbulent systems, for which the fluctuations are much more complex than gaussian delta-correlated noise. The angle $\alpha / 2=1.3$ was chosen as a typical value (quantitatively identical regimes were obtained for values of $\alpha / 2$ in the range $[\pi / 8 ; \pi / 2])$.

We first consider the influence of the standard deviation of the driving $\tilde{\mathcal{A}}$ for a fixed time averaged value $\langle\mathcal{A}\rangle$ and fixed interacting distance $(d=45 \mathrm{~mm})$. Let us first investigate the case where the driving fluctuations are kept very small (i.e. small values of $\tilde{\mathcal{A}}$ ). The interacting magnets then fluctuate around their stable stationary fixed points and typical time series of $\theta_{1}(t)$ and $\theta_{2}(t)$ are given in figure $5(\mathrm{a}-\mathrm{b})$. The associated $\left(\theta_{1}(t), \theta_{2}(t)\right)$ configuration space is displayed in figure $5(\mathrm{c})$, and shows that the dynamics is confined to the potential well centered on $\left(\theta_{1} \sim 0.6, \theta_{2} \sim-0.33\right)$. The trajectory of the fluctuating system is confined to an island in the configuration space and no reversals are observed. This results in a constant large scale dipolar magnetisation of the system, with fluctuations around the time-average dipole strength. The amplitude of the noise is large enough to explore locations in the configuration space where the potential is non zero close to stable fixed point (black circle), but it is too weak to drive the system toward the nearest unstable fixed points (white crosses). For higher values of the driving noise amplitude $\tilde{\mathcal{A}}$, the dynamics is more complex and reversals of the magnets orientation can be observed. Typical time series of $\theta_{1}(t)$ and $\theta_{2}(t)$ in this regime are given in figure $5(\mathrm{~d}-\mathrm{e})$. The system now explores regions close to the unstable fixed points. This is evidenced when investigating the dynamics of the reversals displayed in figure $5\left(\mathrm{~d}\right.$-e) in the $\left(\theta_{1}(t), \theta_{2}(t)\right)$ configuration space as given in figure $5(\mathrm{f})$. In this situation, the noise amplitude is strong enough to drive the system close to the unstable fixed points (white crosses), eventually leading to reversals of the magnets orientations. The trajectory is no more confined to an island. The system explores potential wells around the stable fixed points (black circles) during long intervals, leading to dense trajectories in the vicinity of these stable fixed points. However, the noise is strong enough to drive the system close to the unstable fixed points for short periods of time, before the trajectory is confined again close to a new stable fixed point. From figures 5(c) and (f), it is also clear that the dynamics is confined to valleys of low potential values darker regions - as schematically explained in figure 2(b). This dynamics results in the occurrence of two opposite large-scale dipolar magnetisations (i.e. the dipolar structure can be oriented towards the $x$ direction or the $-x$ direction), with stochastic reversals between these two states. A video displaying the dynamics of such regimes is provided as a supplementary material at [URL will be inserted by publisher]. It shows the view of the magnets (where the red dot locates the north pole of the magnet), together with the time-evolution of angular positions $\theta_{1}$ and $\theta_{2}$ and of the $x$-component of the magnetic field, measured with a Hall effect sensor halfway between the two magnets axes.
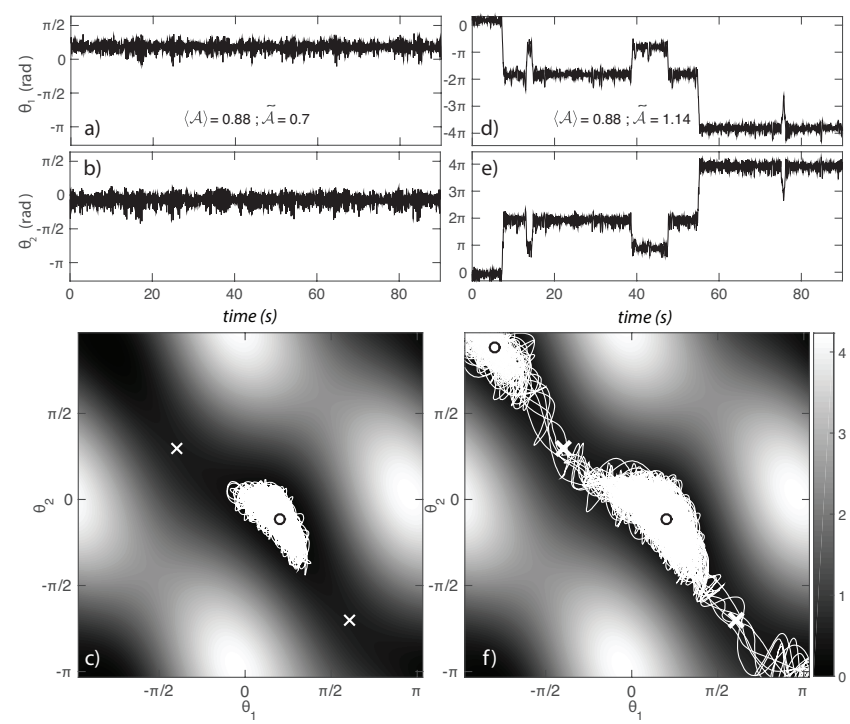

FIG. 5. (a,d) $\theta_{1}(t)$ time series and (b,e) associated $\theta_{2}(t)$ time series for $\alpha / 2=1.3$. (c,f) Corresponding trajectory in configuration space (gray scale codes for the amplitude of potential $V)$. Left column: statistically stationary regime, Right column: reversals. See text for details.

Let us now investigate in greater details the evolution of the dynamics as a function of both the time-averaged value $\langle\mathcal{A}\rangle$ and the standard deviation $\tilde{\mathcal{A}}$ of the normalized driving torque. The experimental evolution of the timeaveraged angular position $\left\langle\theta_{1}\right\rangle$ of magnet 1 as a function of $\langle\mathcal{A}\rangle$ is displayed as dots in figure 6(a) - an evolution in very good agreement with the stable fixed points computed from system (3)-(4) plotted as a full black line. The evolution of $\tilde{\theta_{1}}$, the standard deviation of the fluctuations of the angular position of magnet 1 around $\left\langle\theta_{1}\right\rangle$ is displayed in figure $6(\mathrm{~b})$ as a function of $\langle\mathcal{A}\rangle$ for several values of the driving fluctuations $\tilde{\mathcal{A}}$. Let us first examine the case for which the amplitude of the noise is weak enough so that the dynamics is confined to potential wells around the fixed points in the $\left(\theta_{1}, \theta_{2}\right)$ configuration space (as in figure $5(\mathrm{c})$ ). This is for instance the case for the green (vertical) crosses in figure $6(\mathrm{~b})$, for which $\tilde{\mathcal{A}}=0.35$. The standard deviation $\tilde{\theta_{1}}$ first increases when $\langle\mathcal{A}\rangle$ is increased up to 0.66 and then decreases for higher values of $\langle\mathcal{A}\rangle$. Similar behaviours are observed for higher values of $\tilde{\mathcal{A}}$ : the evolution of $\tilde{\theta_{1}}$ normalised by $\tilde{\mathcal{A}}$ nearly collapse onto a single curve as shown in the inset of figure $6(\mathrm{~b})$. We provide a phenomenological explanation of these features in next section. In this regime, the interacting magnets fluctuate around their mean angular positions $\left\langle\theta_{1}\right\rangle$ and $\left\langle\theta_{2}\right\rangle$ without reversals. For higher values of the driving noise amplitude $\tilde{\mathcal{A}}$, we already mentioned more com- 
plex dynamics, with magnets orientation reversals. For sufficiently large driving noise, the system may explore regions close to the unstable fixed points (displayed in figure 6(a) as a dashed line). These reversals are only observed for a narrow range of $\langle\mathcal{A}\rangle$ values, displayed as arrows in figure $6(\mathrm{~b})$. We will describe in details the case for which $\tilde{\mathcal{A}}=0.79$ - purple squares in figure $6(\mathrm{~b})$. For values of $\langle\mathcal{A}\rangle$ up to 0.7 , the fluctuations of the angular position $\tilde{\theta_{1}}$ remain below $3 \pi / 16$ and the regimes correspond to statistically stationary regimes (with magnets fluctuating around their mean angular positions). For higher values of $\langle\mathcal{A}\rangle$ (between 0.75 and 1.2), stochastic reversals of the magnets orientation are observed. In this regime, the angular positions switches randomly between two angular positions - with a large level of fluctuations. These positions correspond to the stationary fixed points and each reversal consist in a rapid rotation of angle $n \pi$, $n$ being an unsigned integer. The extent of these regimes is depicted as a full arrow in figure 6(b) (since reversals of the magnets occur, the standard deviation is no longer an illustrative variable to consider for these regimes). Then, as $\langle\mathcal{A}\rangle$ is further increased, the system do not exhibit reversals any longer and the system recovers a statistically stationary dynamics, with a decreasing evolution of $\theta_{1}$ with $\langle\mathcal{A}\rangle$.
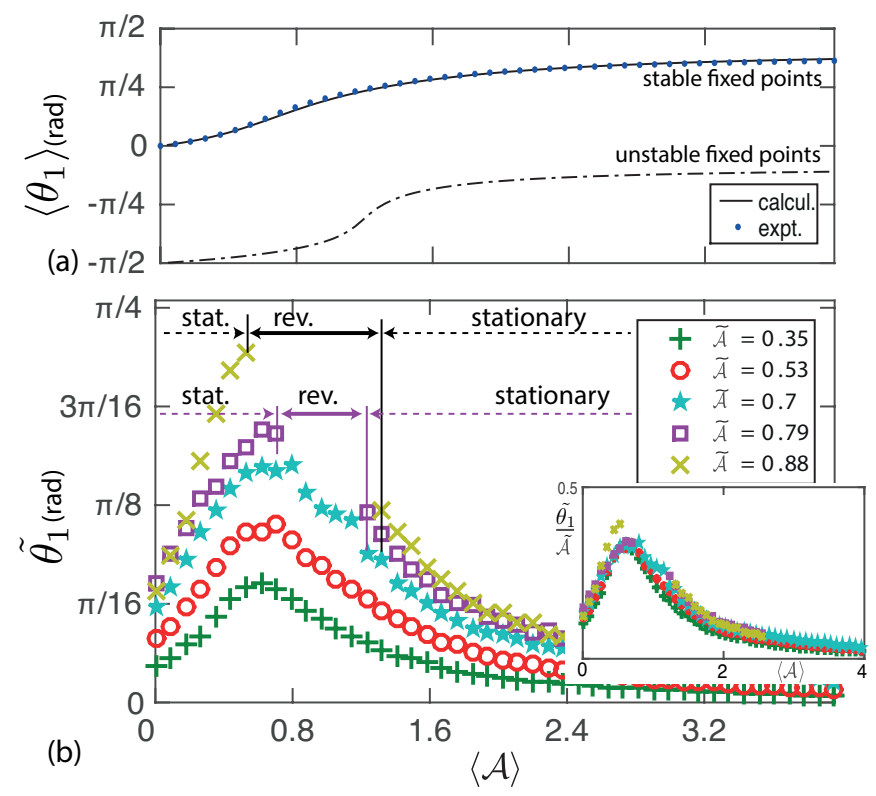

FIG. 6. (a) Evolution of the time average value $\left\langle\theta_{1}\right\rangle$ : (full line) stable fixed points and (dash-dotted) line unstable fixed points computed from equations 3-4 and experiments (circles) and (b) evolution of the standard deviation $\tilde{\theta_{1}}$ as a function of $\langle\mathcal{A}\rangle$ for various $\tilde{\mathcal{A}}$ values. The distance is kept constant $d=45 \mathrm{~mm}$ and $\alpha / 2=1.3$.

The first observation is that stochastic reversals can be observed with a gaussian white noise driving if the noise amplitude is sufficiently large. A second observation, is that the extent of the occurrence of these reversals depends on the driving noise amplitude.
A third observation is that, close to the onset of existence of stochastic regimes - i.e. for the smallest noise amplitude where reversals have been observed - these regimes are bounded by statistically stationary regimes as a function of $\langle\mathcal{A}\rangle$ when $\tilde{\mathcal{A}}$ is kept constant.

The determination of the extent of the regimes where reversals are observed as depicted in figure 6(b) is somewhat abritrary. This can be best understood from the analysis of figure 7(a) which displays the evolution of the mean waiting time $\left\langle T_{r}\right\rangle$ between reversals as a function of $\langle\mathcal{A}\rangle$ for a fixed value of $\tilde{\mathcal{A}}=1.14$ and $d=45 \mathrm{~mm}$. For these parameters, the situation is similar to the one presented in figure 6(b): reversals are bounded by statistically stationary regimes. Data presented in figure 7(a) where obtained from 86400 seconds (24 hours) long time series. The first observation is that reversals are more frequent in the center of the occurrence region than at its boundary. The second observation is that the mean waiting time between reversals rapidly increases as $\langle\mathcal{A}\rangle$ is decreased or increased to values corresponding to the statistically stationary regimes. Whether the mean waiting time between reversals diverges or not as $\langle\mathcal{A}\rangle$ approaches the values for which no reversals occur cannot be stated definitely from these data (this would require extremely long time series), but divergence is compatible with the evolution depicted in figure 7(a). Boundaries between regimes should thus be defined as when the mean time between reversals diverges. However, given the evolution provided in figure $7(\mathrm{a})$, a precise determination of boundaries between regimes in the parameter space $(\langle\mathcal{A}\rangle, \tilde{\mathcal{A}})$ for various values of $d$ is a rather difficult task (which would require a very large number of extremely long time series - a task out of reach of this study). We provide in figure $7(\mathrm{~b})$ an estimate of these boundaries from the analysis of finite length time series (at least two reversals over 480 seconds long time series) for three values of the coupling distance $d$ and a fine scan in the $(\langle\mathcal{A}\rangle, \tilde{\mathcal{A}})$ parameter space. Let us first focus on the case $d=45 \mathrm{~mm}$, which corresponds to the (blue) squares. In this figure, the lower and upper threshold in $\langle\mathcal{A}\rangle$ for the occurrence of stochastic reversals are plotted as a function of $\tilde{\mathcal{A}}$-this corresponds to the extent of the full arrows in figure 6(b). Schematically, three distinct regimes are observed for a given amplitude of the driving noise (equivalently $\tilde{\mathcal{A}}$ ): (i) for the lowest values of the driving noise, only statistically stationary regimes are observed (left hand side of figure $7(\mathrm{~b})$ ), (ii) for moderate amplitude of the driving noise, reversals are bounded by statistically stationary regimes as $\langle\mathcal{A}\rangle$ increases, (iii) for large amplitude of the driving noise, reversals are observed when $\langle\mathcal{A}\rangle$ is below a threshold, while statistically stationary regimes are observed above. We provide a phenomenological explanation of this sequence in next section. The influence of the coupling strength on the occurrence of the stochastic regimes is also displayed in figure $7(\mathrm{~b})$, for two other values of the coupling distance $d$, namely $40 \mathrm{~mm}$ (black stars) and $55 \mathrm{~mm}$ (empty red circles). A first striking 
observation is that the onset of stochastic regimes is observed for lower values of the noise when the coupling is stronger. A second observation is that for a given value of the noise amplitude, the window of occurence for theses regimes is smaller for stronger coupling. Finally, the time statistics of waiting time between reversals is studied through their probability distribution functions (pdf) displayed in figure 7(c). These pdf have been computed from 259200 seconds long (72 hours) time series for $\langle\mathcal{A}\rangle=0.97$ and 324000 seconds long (90 hours) for the other two values of $\langle\mathcal{A}\rangle$. These pdf are compatible with an exponential distribution on a first approximation, with characteristic times (or equivalently meanwaiting times between reversals) $\tau=56.2 ; 12.1$ and 26.3 $\mathrm{s}$ respectively for $\langle\mathcal{A}\rangle=0.44,0.97,1.36$.

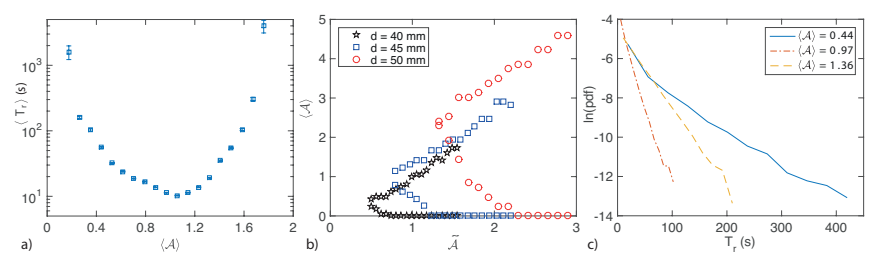

FIG. 7. (a) Evolution of the mean waiting time between reversals $\left\langle T_{r}\right\rangle$ as a function of $\langle\mathcal{A}\rangle$, for $\tilde{\mathcal{A}}=1.14$. (b) Estimated boundaries between regimes in the $(\tilde{\mathcal{A}},\langle\mathcal{A}\rangle)$ parameter space for $\alpha / 2=1.3$. (c) Probability density function of waiting times between reversals for $\tilde{\mathcal{A}}=1.14$, and $d=45 \mathrm{~mm}$ for the three values of $\langle\mathcal{A}\rangle$. See text for details.

\section{PHENOMENOLOGICAL MODEL}

In this section, we analyze the features of the coupled stochastic system in a simplified framework which explains qualitatively most of the experimental observations. This analysis relies on the interpretation of the potential energy $V$ introduced earlier. In the presence of fluctuations of the driving torque, the potential $V$ can then be written as the sum of two contributions: a time averaged potential $\langle V\rangle$ and a noise $\eta$.

$$
V(t)=\langle V\rangle+\eta(t)
$$

where

$$
\langle V\rangle=-3 \cos \theta_{1} \cos \theta_{2}+\cos \left(\theta_{2}-\theta_{1}\right)+\frac{\langle\mathcal{A}\rangle}{2} \cos \left(\alpha-2 \theta_{1}\right)+C,
$$

and

$$
\eta(t)=\frac{\mathcal{A}(t)-\langle\mathcal{A}\rangle}{2} \cos \left(\alpha-2 \theta_{1}\right)
$$

Let us now make the approximation that the energy landscape is set by the time-average value of the potential $\langle V\rangle$, while the fluctuations of the driving act as a noise which allows to reach locations of the configuration space where the potential is positive. In the real system, fluctuations of the driving indeed modify the instantaneous energy landscape, but, for the sake of simplicity, we neglect these modifications at leading order. Under our approximation, trajectories in the $\left(\theta_{1}, \theta_{2}\right)$ configuration space evolve within the time average potential $\langle V\rangle$. We assume that excursions in the $\left(\theta_{1}, \theta_{2}\right)$ configuration space are restricted to locations where the value of the mean potential is lower than a maximal value proportional to $\tilde{\eta}$ where $\tilde{\eta}$ is the standard deviation of the noise. The evolution of isocontours of the potential as a function of $\langle\mathcal{A}\rangle$ thus provide useful insight of the expected dynamics of the system; these are displayed in figure 8 . For each value of $\langle\mathcal{A}\rangle$, three isolines are represented, respectively 0.5 (full line), 0.66 (dash-dotted line) and 1.01 (dotted line). We will assume that these isocontours represent the accessible locations in the $\left(\theta_{1}, \theta_{2}\right)$ configuration space for increasing values of the driving noise. The analysis of each of the three cases allows for providing a phenomenological understanding of the stationary and reversals regimes, their evolutions and the transitions between them as described in figure 7(b).

We will first focus on the evolution of isocontours for potential $\langle V\rangle=0.5$ (full lines). For all values of $\langle\mathcal{A}\rangle$, isocontours separate islands around a stable fixed point. The noise is not strong enough to drive the system close to an unstable fixed point: the dynamics of the system is restricted to fluctuations around one of the stable fixed points. This is the situation encountered when $\tilde{\mathcal{A}}<0.7$ in figure 6: no reversals are observed whatever the value of $\langle\mathcal{A}\rangle$. The trends observed in figure 6(b) for the evolution of $\tilde{\theta_{1}}$ (namely increasing with $\langle\mathcal{A}\rangle$ at low values and decreasing with $\langle\mathcal{A}\rangle$ at large values) can also be understood from the evolution of the isocontours of the potential landscape. Indeed, the extent of the islands - and thus the accessible range of $\theta_{1}$ values - first increases with $\langle\mathcal{A}\rangle$ (at low values), and then decreases with $\langle\mathcal{A}\rangle$ (for larger values). Let us now consider the isocontours for potential 0.66 (dash-dotted lines). For $\langle\mathcal{A}\rangle$ values below 0.4 and above 1.3, the potential isocontours separate islands around stable fixed points: no reversals are observed (similarly to the evolution depicted in figure $6(\mathrm{~b})$ for $\tilde{\mathcal{A}}=0.88$ at the lowest and highest values of $\langle\mathcal{A}\rangle)$. On the contrary, when $0.4 \leq\langle\mathcal{A}\rangle \leq 1.3$, isocontours encompass both stable fixed points. In this $\langle\mathcal{A}\rangle$ interval, the noise amplitude is strong enough to explore vicinities of the unstable fixed points and leads to reversals of the magnets orientation. Once again, the evolution of $\tilde{\theta_{1}}$ as a function of $\langle\mathcal{A}\rangle$ for the stationary regimes can be understood from the extent of the isocontour islands. Finally, isocontours for potential 1.01 (dotted lines) show a connexion between stable fixed points as long as $\langle\mathcal{A}\rangle$ is below 1.7, allowing for reversals of the magnets orientations. For values of $\langle\mathcal{A}\rangle$ above 1.7, the isocontours separate islands around stable fixed points, and statistically stationary regimes are recovered. While the direct computation of the relation between the amplitude of 

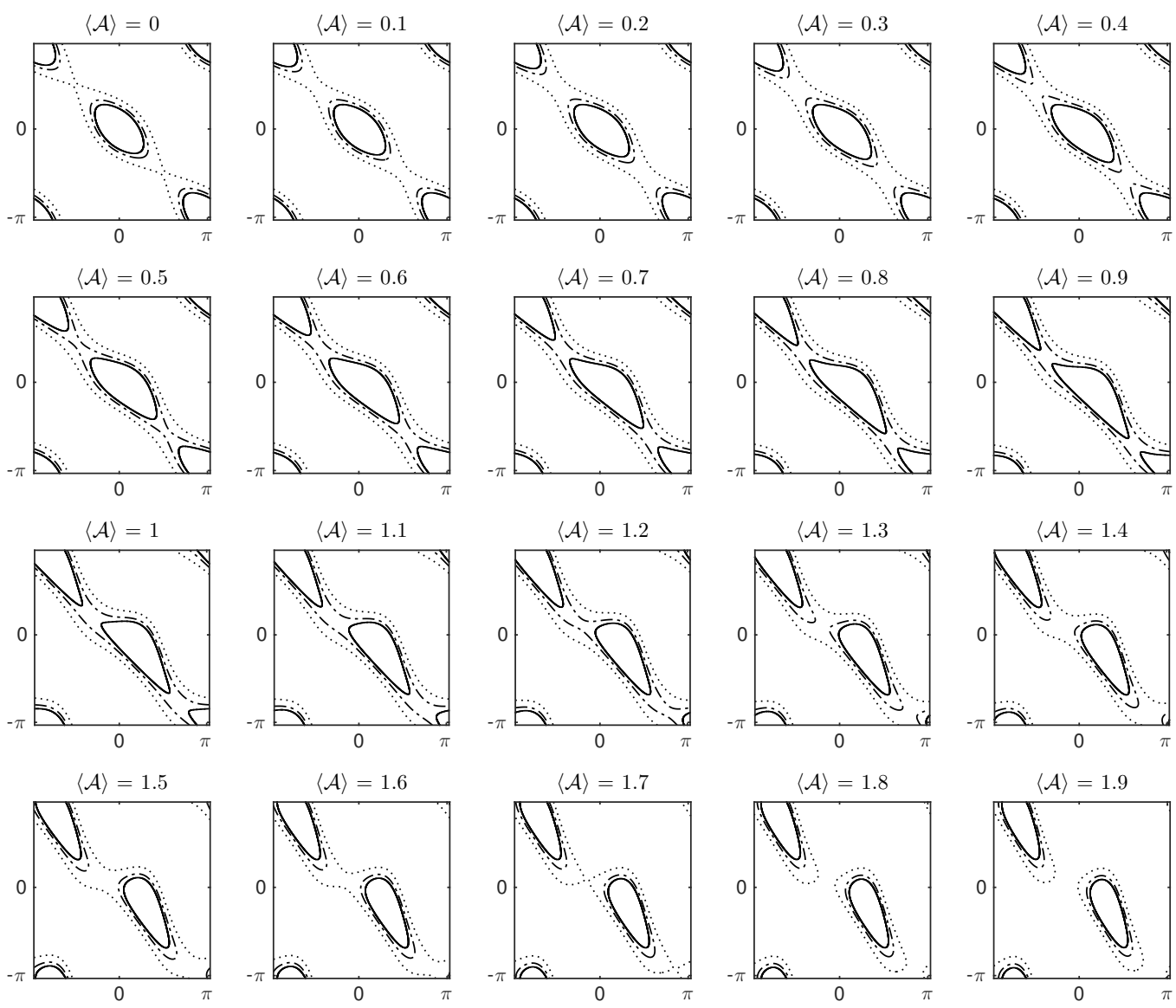

FIG. 8. Isolines of $V$ for increasing values of $\langle\mathcal{A}\rangle$ in the configuration space $\left(\theta_{1}, \theta_{2}\right)$ from top left to bottom right. Isopotentials 0.5 (full lines), 0.66 (dash-dotted lines) and 1.01 (dotted lines). See text for details.

the fluctuations of the driving current $\tilde{I}_{0}$ and the maximum potential value accessible to the system is a difficult task, the above rationale gives an accurate understanding of the observed dynamics and reproduces the three regimes described above, as well as the evolution of $\tilde{\theta_{1}}$ in the $(\langle\mathcal{A}\rangle, \tilde{\mathcal{A}})$ parameter space. Regime (ii) described above (namely that for moderate values of the driving noise, reversals are bounded by statistically stationary regimes when modifying the time-averaged value of the driving) can be understood the following way: for intermediate values of $\langle\mathcal{A}\rangle$, adding a driving torque to magnet 1 strongly modifies the energy landscape and lowers the energy gap between stable and unstable fixed points and eases the observation of reversals.

\section{DISCUSSION AND CONCLUSION}

We reported the rich dynamics displayed by a simple table-top experiment involving the coupling of two macroscopic magnetic dipoles in presence of a driving torque on one of the magnet - which preserves a $\pi$ rotation symmetry of the fixed points of the system. The accessible fixed points are accurately determined introducing an effective potential accounting for both the coupling and the driving. In the presence of fluctuations of the driving torque, two distinct behaviours are observed: the dipoles fluctuate around their stable fixed points (typically for low amplitudes of the fluctuations) or they can display stochastic reversals between stable fixed points (when fluctuations are strong enough to drive the system close to unstable fixed points). The mapping of these two regimes in the parameter space is accurately described when considering an energy argument: the dynamics is then restricted to locations in the configurationspace for which the effective potential energy is lower than a threshold (related to the energy of the fluctuations). For low energy of the fluctuations, the dynamics is restricted to disjoint basins of attraction close to the stable fixed points. For higher fluctuation energies, 
these basins become connected (now including an unstable fixed point) and the dynamics displays stochastic reversals with Poisson-distributed waiting time between reversals.

Our table-top experiment was built somehow in the spirit of the one in [11]. It could be considered as a building block of a macroscopic experiment of a finite number of interacting dipoles, as reported numerically in $[3,4]$. Our driving scheme preserves symmetries and could be used to mimic defects in the lattice and/or noise mimicking thermal fluctuations. It could also be used as a macroscopic benchmark for the dynamical studies of slow-relaxing low-dimensional magnetic systems, such as one dimensional single-chain magnets [12].

The present experiment has also been designed to develop a simple model of the dynamics of chaotic reversals of the turbulent liquid metal dynamo experiment VKS (Von Kármán Sodium) - in which liquid sodium is set into motion in a cylindrical container by the rotation of coaxial impellers fitted with blades. Under specific parameters, the observed magnetic field can exhibit complex dynamics, including random and sudden reversals between opposite polarities [13]. A model has been proposed [14] which explains the occurrence of such reversals as a stochastic transition (activated by the turbulent fluctuations of the flow) between two large scale magnetic modes. In turn, these large scale modes (dipole and quadrupoles) and their stability properties can be understood from the interaction between dipoles localised in the vicinity of each ferromagnetic impeller (such as two parallel or anti-parallel localised dipoles) [15]. The tabletop experiment described in the present article was inspired by this interpretation: each of the magnet dipole represents one of the localised dynamo, and the timedependent driving models the influence of large-scale turbulent fluctuations (which are modeled by gaussian white noise). The present experiment shares very similar behaviours with the ones observed in the VKS experiment, such as (i) existence of pockets of dynamical regimes bounded by stationary regimes [16], (ii) Poisson distribution of the waiting time between reversals [8], (iii) divergence of the waiting time between reversals at the transition between stationary and dynamical regimes. This setup thus allows to understand, in a very simplified framework, the relative influence of the coupling between the two localised modes and the fluctuations of the driving on the occurrence of reversals. In particular, we showed a counter-intuitive result: a higher level of fluctuations is required to obtain reversals when the coupling strength between the two magnets decreases (i.e. the coupling distance increases). However, keeping in mind the comparison between the present work with the actual MHD system, several points are worth being mentioned : (i) in the present system, the localised dipoles have constant amplitude and are only allowed to rotate, whereas in the case of the actual MHD dynamo, the amplitude also fluctuates; (ii) the noise mimicking turbulent fluctuations is imposed here as gaussian white noise and only on one of the dipoles. Our results show that in spite of its simplicity and apparent naiveness, this simple system reproduces correctly some basic dynamical features of the magnetic field in VKS.

Evolution of this simple experiment could also be implemented in views of providing quantitive understanding of other stochastic phenomena. For instance, a spontaneous bifurcation between symmetric and and asymmetric flow configurations have been reported in fully turbulent vonKarman flows subject to multistability [5]. Similar bifurcations might be observed in our simple table-top experiment when driving both magnets with independent stochastic torques and would open ways to provide simple analysis of the dynamics of complex systems.

\section{ACKNOWLEDGMENTS}

We thank M. Moulin for his technical assistance, D. Micard for her participation in preliminary experiments, and G. Verhille for fruitful discussions.

\section{Appendix A: System Differential equation}

\section{Torque applied to shaft 1 by the drive}

In this appendix, the system of differential equations describing the coupled dynamics of the rotating shafts is determined. Let us first describe the driving scheme. It consists of a quadrupolar permanent magnet fixed on shaft 1 upon which a torque is imposed by an external quadrupolar magnetic field created by current circulation in 8 coils arranged around the quadrupolar permanent magnet (see Figure 1 of the mauscript). The quadrupolar permanent magnet, of magnetic moment $\mathcal{M}_{q}$ is aligned with the dipolar moment $\mathcal{M}_{1}$ (if $\theta_{1}=0$, the outgoing directions of the magnetic field-lines from the permanent quadrupolar magnet are 0 and $\pi$, relative to axis $x$ ). It is assembled from 4 small dipolar cylindrical segments magnets of moment $0.354 \mathrm{Am}^{2}$. The 8 coils create a magnetic field $\mathbf{Q}_{\text {coils }}$, of amplitude $Q_{\text {coils }}$ and whose outgoing field lines are oriented at an angle $\alpha / 2$ (modulus $\pi$ ) relative to the $x$ axis. This is achieved by driving currents $I_{i}$ in coil $i$ as $I_{1}=I_{5}=-I_{3}=-I_{7}=I_{0} \cos \alpha$ and $I_{2}=I_{6}=-I_{4}=-I_{8}=I_{0} \sin \alpha$. The strength of the externally applied magnetic field $Q_{\text {coils }}$ is then proportional to the current $I_{0}$. The angle $\alpha / 2$ sets a preferential orientation for magnet 1 (reached when $I_{0} \rightarrow \infty$ for finite d). The driving torque applied by the external magnetic quadrupolar field $\mathbf{Q}_{\text {coils }}$ on the quadrupolar permanent magnet reads

$$
\Gamma_{\text {drive }}=Q_{\text {coils }} \mathcal{M}_{q} \sin \left(2\left(\alpha / 2-\theta_{1}\right)\right)=D I_{0} \sin \left(\alpha-2 \theta_{1}\right)
$$

With $D$ an effective driving efficiency accounting for the coupling between the quadrupolar permanent magnetic field and the applied quadrupolar magnetic field. 


\section{Torque applied to shaft 1 by the drive}

The full mechanical system is obtained when applying Newton's laws to each of the two shafts.

Torque applied by magnet 2 on magnet 1 reads

$\Gamma_{21}=\mathcal{M}_{1} \times B_{2}=-\frac{\mu_{0} \mathcal{M}_{1} \mathcal{M}_{2}}{4 \pi d^{3}}\left(\sin \theta_{2} \cos \theta_{1}+2 \sin \theta_{1} \cos \theta_{2}\right)$

Similarly, the torque applied by magnet 1 on magnet 2 reads

$\Gamma_{12}=\mathcal{M}_{2} \times B_{1}=-\frac{\mu_{0} \mathcal{M}_{1} \mathcal{M}_{2}}{4 \pi d^{3}}\left(\sin \theta_{1} \cos \theta_{2}+2 \sin \theta_{2} \cos \theta_{1}\right)$

With $J_{i}, F_{i}$ and $K_{i}$ respectively the moments of inertia of shaft $i$, the fluid friction coefficient and the solid friction coefficient acting on shaft $i$, the coupled dynamics of the system is then described by the set of 2 differential equations (A1, A2)

$$
\begin{aligned}
J_{1} \frac{d^{2} \theta_{1}}{d t^{2}}= & D I_{0} \sin \left(\alpha-2 \theta_{1}\right)-\frac{\mu_{0} \mathcal{M}_{1} \mathcal{M}_{2}}{4 \pi d^{3}}\left(\sin \theta_{2} \cos \theta_{1}\right. \\
& -F_{1} \frac{d \theta_{1}}{d t}-K_{1} \operatorname{sign}\left(\frac{d \theta_{1}}{d t}\right), \\
J_{2} \frac{d^{2} \theta_{2}}{d t^{2}}= & -\frac{\mu_{0} \mathcal{M}_{1} \mathcal{M}_{2}}{4 \pi d^{3}}\left(\sin \theta_{1} \cos \theta_{2}+2 \sin \theta_{2} \cos \theta_{1}\right) \\
& -F_{2} \frac{d \theta_{2}}{d t}-K_{2} \operatorname{sign}\left(\frac{d \theta_{2}}{d t}\right),
\end{aligned}
$$

\section{Appendix B: Parameters identification}

All parameters of the differential system $(\mathrm{A} 1, \mathrm{~A} 2)$ have been directly measured or identified from the dynamics of the uncoupled system (i.e. with an infinite $d$ or with one of the shaft kept static), which simplifies as the dynamics of a damped second order system.

a. Measurement of $\mathcal{M}_{i}: \quad \mathcal{M}_{1}$ and $\mathcal{M}_{2}$ have been computed by fitting the evolution of the magnetic field created by each small dipolar magnet as a function of the distance $z$ along the axis of magnetisation from the center of the magnet. The theoretical calculation gives $B=\frac{\mu_{0} \mathcal{M}_{1}}{2 \pi z^{3}}$. The logarithmic evolution is linear for $z>$ $25 \mathrm{~mm}$ and gives $\mathcal{M}_{1}=\mathcal{M}_{2}=0.775 \mathrm{Am}^{2}$.

b. Measurement of $J_{i}, F_{i}, K_{i}$ and $D$ : Mechanical parameters were estimated from the relaxation dynamics of uncoupled or coupled shafts in the presence of a magnetic field.

1) A first set of measurements can be performed in the presence of a uniform, static magnetic field. Three different configurations were studied:

- A uniform, static, dipolar magnetic field $B_{\text {out }}$ is applied from external large electrical coils in a Helmholtz configuration along the reference horizontal axis around the small permanent magnet, for one single shaft. The uncoupled dynamics of shaft $i$ is then

$$
J_{i} \frac{d^{2} \theta_{i}}{d t^{2}}=-\mathcal{M}_{1} B_{\text {out }} \sin \theta_{i}-F_{i} \frac{d \theta_{i}}{d t}-K_{i} \operatorname{sign}\left(\frac{d \theta_{i}}{d t}\right)
$$

- When shaft $j$ is maintained at a fixed angular position $\theta_{j}=0$, a static dipolar magnetic field $B_{j}$ (created by the permanent magnet $j$ ) is applied to the small permanent magnet on shaft $i$. The system considered here is the one displayed in figure 1, for which one of the two shaft is set static. The dynamics of shaft $i$ is then

$$
J_{i} \frac{d^{2} \theta_{i}}{d t^{2}}=-\frac{\mu_{0} \mathcal{M}_{1} \mathcal{M}_{2}}{2 \pi d^{3}} \sin \theta_{i}-F_{i} \frac{d \theta_{i}}{d t}-K_{i} \operatorname{sign}\left(\frac{d \theta_{i}}{d t}\right)
$$

- A static driving quadrupolar magnetic field is applied from current circulation in the 8 driving coils. When $\alpha=0$, the dynamical equation for the uncoupled dynamics of shaft 1 is then

$$
J_{1} \frac{d^{2} \theta_{1}^{2}}{\left.d t^{2} 1\right)}=D I_{0} \sin \left(-2 \theta_{1}\right)-F_{1} \frac{d \theta_{1}}{d t}-K_{1} \operatorname{sign}\left(\frac{d \theta_{1}}{d t}\right)
$$

For all these configurations, the dynamics of a single shaft is described by a second order differential equation with damping. The temporal evolution of the relaxation from $\theta_{i} \neq($ Ais $)$ then recorded for various values of the magnetic field setting the resonance frequency (i.e. varying either $B_{\text {out }}, I_{0}$ or $d$ for the three cases specified above). An estimate of the linear relaxation pulsations $\sqrt{\frac{\mathcal{M}_{i} B_{\text {out }}}{J_{i}}}$, $\sqrt{\frac{\mu_{0} \mathcal{M}_{1} \mathcal{M}_{2}}{2 \pi d^{3} J_{i}}}$ or $\sqrt{\frac{2 D I_{0}}{J_{i}}}$ is computed from the temporal evolution - see for instance figure 9(a) for a typical relaxation of uncoupled shaft 1 with an applied $B_{\text {out }}$ around magnet 1 . An estimate of the fluid friction coefficient $F_{i}$ is provided by the temporal evolution of $\ln \left(\theta_{i}\right)$ (whose envelope is linear with time at initial stages - see the inset figure 9(a)). Eventually, the solid friction coefficient $K_{i}$ is estimated from the latest stage of the temporal evolution of the envelope of $\theta_{i}$, which is linearly decreasing at latest times - see figure 9(a). From these first-guess estimates, a least square fitting method is applied between the experimental relaxation and numerical integration of equations (3), (4) or (5). An example of the comparison between the experimental data and the best fit obtained is given in figure $9(\mathrm{a})$.

2) The determination of the coefficients is also made possible when driving uncoupled shaft 1 with a constant driving current $I_{0}$ and an harmonic variation of $\alpha=2 A \cos (2 \pi f t)$ (shaft 1 has to be uncoupled from shaft 2 , i.e. $d \rightarrow \infty)$. In that case the dynamics is governed by

$$
J_{1} \frac{d^{2} \theta_{1}}{d t^{2}}=D I_{0} \sin \left(2 A \cos (2 \pi f t)-2 \theta_{1}\right)-F_{1} \frac{d \theta_{1}}{d t}-K_{1} \operatorname{sign}\left(\frac{d \theta_{1}}{d t}\right)
$$



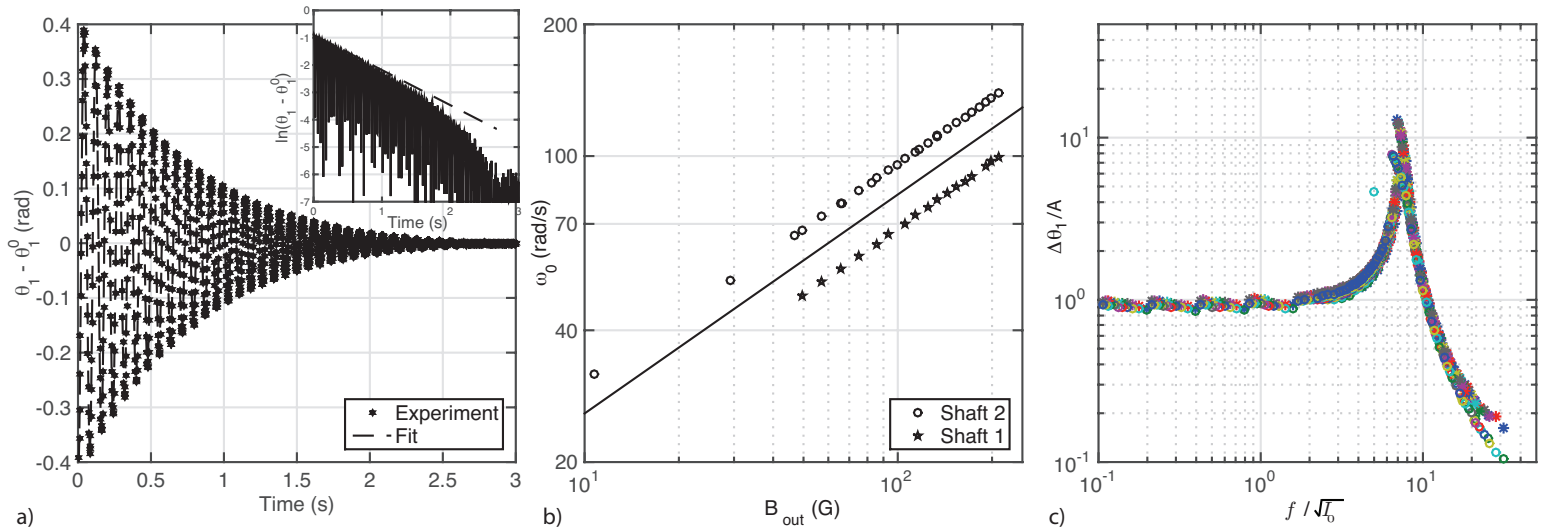

FIG. 9. a) Dynamics of the relaxation for shaft 1 (uncoupled and in the presence of an external uniform magnetic field $B_{\text {out }}=144 \mathrm{G}$ ). Experimental point and non-linear fit for the estimate of parameters. Inset: same graph in logarithmic scale. See text for details. b) Evolution of the resonant pulsation $\omega_{0}$ as a function of $B_{\text {out }}$ for both shafts. See text for details. c) $\Delta \theta_{1} / A$ as a function of $f / \sqrt{I_{0}}$ for 10 values of $I_{0}$ (from 0.5 to $1.4 \mathrm{~A}$ in steps of $0.1 \mathrm{~A}$ ) and 2 values of $A(0.05$ and $0.1 \mathrm{rad}$ )

The evolution of the amplitude of the response $\Delta \theta_{1}$ is typical of a resonant second-order system, with a static - low frequency - response $\Delta \theta_{1}$ equal to $A$ and a resonance giving access to the resonance frequency and to the damping. Typical evolutions of $\Delta \theta_{1}$ as a function of $f / \sqrt{I_{0}}$ are displayed in figure $9(\mathrm{c})$.

From these methods, the following values for the coefficient have been measured:

$$
\text { - } \mathcal{M}_{1}=\mathcal{M}_{2}=0.775 \mathrm{~A} . \mathrm{m}^{2} \text {. }
$$

- $J_{1}=1.6410^{-6} \mathrm{~kg} \cdot \mathrm{m}^{2}$, and $J_{2}=8.310^{-7} \mathrm{~kg} \cdot \mathrm{m}^{2}$, values compatible with rough computation from geometrical considerations.

- $D=1.4510^{-3}$ T.m ${ }^{2}$.

- $F_{1} / J_{1} \sim F_{2} / J_{2} \sim 2.2 \mathrm{~s}^{-1}$.

- $K_{1} / J_{1} \sim 2.8 \mathrm{~s}^{-2}$ and $K_{2} / J_{2} \sim 7.5 \mathrm{~s}^{-2}$.
[1] Van Kampen, N.G., "Stochastic Processes in Physics and Chemistry" Elsevier, ISBN: 978-0-444-52965-7, (2007)

[2] Kramers, H. A. "Brownian motion in a field of force and the diffusion model" Physica, 7, (4), 284-304, (1940)

[3] Borgonovi, F., Celardo, G. L. "Dynamics of random dipoles: chaos versus ferromagnetism." J. Stat. Mech., 05, P05013, (2010)

[4] Celardo, G. L., Barré J., Borgonovi, F., Ruffo, S. "Time scale for magnetic reversal and the topoligical nonconnectivity threshold." Phys. Rev. E, 73, 011108, (2006)

[5] Ravelet, F., Marié, L., Chiffaudel, A., Daviaud, F., "Multistability and Memory Effect in a Highly Turbulent Flow: Experimental Evidence for a Global Bifurcation" Physical Review Letters, 93 (16), 164501, (2004)

[6] De la Torre, A., Burguete, J., "Slow dynamics in a turbulent von Kármán swirling flow", Physical Review Letters, 99, 054101, (2007)

[7] Machicoane, N., Zimmermann, R., Fiabane, L., Bourgoin, M., Pinton, J.-F., Volk, R. "Large sphere motion in a nonhomogeneous turbulent flow." New Journal of Physics, 16 (1), 013053, (2015)

[8] Monchaux, R., Berhanu, M., Aumaître, S., Chiffaudel, A., Daviaud, F., Dubrulle B., Ravelet, F., Fauve, S., Mordant, N., Pétrélis, F., Bourgoin, M., Odier P., Pinton, J-F., Plihon, N., Volk, R., "The von Kármán Sodium experiment: Turbulent dynamical dynamos", Physics of Fluids, 21, 035108, (2009)
[9] Miralles, S., Plihon, N., Pinton, J.-F. "Lorentz force effects in the Bullard von Kármán dynamo: saturation, energy balance and subcriticality." J. Fluid Mech., 775, 501-523, (2015)

[10] Pollack, G.L. and Stump, D.R. "Two magnets oscillating in each other's fields" Can. J. Phys., 75, 313-324, (1997)

[11] Mellado, P., Concha, A., Mahadevan, L. "Macroscopic magnetic frustration." Phys. Rev. Lett., 109, 257203, (2012)

[12] Zhang, W.-X., Ishikawa, R., Breedlovea, B., Yamashita, M. "Single-chain magnets: beyond the Glauber model." RSC Advances, 3, 3772, (2013)

[13] Ravelet, F., Berhanu, M., Monchaux, R., Aumaître, S., Chiffaudel, A., Daviaud, F., Dubrulle, B., Bourgoin, M., Odier, Ph., Plihon, N., Pinton, J.-F., Volk, R., Fauve, S., Mordant, N., Pétrélis "Chaotic Dynamos Generated by a Turbulent Flow of Liquid Sodium." Phys. Rev. Lett., 101, 074502, (2008)

[14] Pétrélis, F., Fauve, S. , "Chaotic dynamics of the magnetic field generated by dynamo action in a turbulent flow." Journal of Physics Condensed Matter, 20, 0 5, (2008)

[15] Verhille, G., Plihon, N., Bourgoin, M., Odier, Ph., Pinton, J.-F. " Induction in a von Kármán flow driven by ferromagnetic impellers" New J. Phys., 12033006 (2010)

[16] Berhanu, M., Verhille, G., Boisson, J., Gallet, B., Gissinger, C., Fauve, S., Mordant, N., Pétrélis, F., Bour- 
goin, M. Odier, Ph., Pinton, J.-F., Plihon, N., Aumaître, S., Chiffaudel, A., Daviaud, F., Dubrulle, B., Pirat, C.
"Dynamo regimes and transitions in the VKS experiment." Eur. Phys. J. B, 77, 459-468, (2010) 